\title{
The non-autonomous YdKN equation and generalized symmetries of Boll equations
}

Cite as: J. Math. Phys. 58, 053507 (2017); https://doi.org/10.1063/1.4982747

Submitted: 24 January 2016 . Accepted: 17 April 2017 . Published Online: 11 May 2017

G. Gubbiotti, C. Scimiterna, and D. Levi (D)

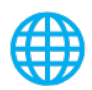

View Online
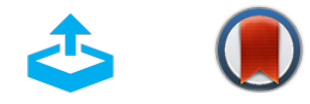

Export Citation

CrossMark

\section{ARTICLES YOU MAY BE INTERESTED IN}

A fundamental solution to the Schrödinger equation with Doss potentials and its smoothness

Journal of Mathematical Physics 58, 053506 (2017); https://

doi.org/10.1063/1.4983132

On cosmological constant of generalized Robertson-Walker space-times

Journal of Mathematical Physics 58, 053508 (2017); https://

doi.org/10.1063/1.4983134

Variable-length analog of Stavskaya process: A new example of misleading simulation

Journal of Mathematical Physics 58, 053304 (2017); https://

doi.org/10.1063/1.4983567

\section{Journal of}

Mathematical Physics

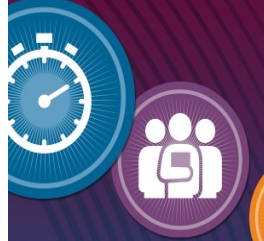

READY TO SUBMIT YOUR RESEARCH?

There are many reasons to publish with us. 


\title{
The non-autonomous YdKN equation and generalized symmetries of Boll equations
}

\author{
G. Gubbiotti, ${ }^{\text {a) }}$ C. Scimiterna, ${ }^{\text {b) }}$ and D. Levic) \\ Dipartimento di Matematica e Fisica, Università degli Studi Roma Tre, e Sezione INFN \\ di Roma Tre, Via della Vasca Navale 84, 00146 Roma, Italy
}

(Received 24 January 2016; accepted 17 April 2017; published online 11 May 2017)

In this paper, we study the integrability of a class of nonlinear non-autonomous quad graph equations compatible around the cube introduced by Boll in the framework of the generalized Adler, Bobenko, and Suris (ABS) classification. We show that all these equations possess three-point generalized symmetries which are subcases of either the Yamilov discretization of the Krichever-Novikov equation or of its non-autonomous extension. We also prove that all those symmetries are integrable as they pass the algebraic entropy test. Published by AIP Publishing. [http://dx.doi.org/10.1063/1.4982747]

\section{INTRODUCTION}

In 1983, Yamilov ${ }^{33}$ classified all differential difference equations of the class

$$
\dot{u}_{n}=f\left(u_{n-1}, u_{n}, u_{n+1}\right)
$$

using the generalized symmetry method. From the generalized symmetry method, one obtains integrability conditions which allow us to check whether a given equation is integrable. Moreover in many cases, these conditions enable us to classify equations, i.e., to obtain complete lists of integrable equations belonging to a certain class. As integrability conditions are only necessary conditions for the existence of generalized symmetries and/or conservation laws, one then has to prove that the equations of the resulting list really possess generalized symmetries and conservation laws of sufficiently high order. One constructs them using Miura-type transformations and master symmetries, proving the existence of Lax pairs. ${ }^{34,35}$ The result of Yamilov classification, up to Miura transformation, are the Toda equation and the so-called Yamilov discretization of the Krichever Novikov equation (YdKN), a differential difference equation depending on 6 arbitrary coefficients

$$
\frac{\mathrm{d} q_{k}}{\mathrm{~d} t}=\frac{A\left(q_{k}\right) q_{k+1} q_{k-1}+B\left(q_{k}\right)\left(q_{k+1}+q_{k-1}\right)+C\left(q_{k}\right)}{q_{k+1}-q_{k-1}},
$$

where

$$
\begin{gathered}
A\left(q_{k}\right)=a q_{k}^{2}+2 b q_{k}+c, \\
B\left(q_{k}\right)=b q_{k}^{2}+d q_{k}+e, \\
C\left(q_{k}\right)=c q_{k}^{2}+2 e q_{k}+f .
\end{gathered}
$$

The integrability of (2) is proven by the existence of point symmetries ${ }^{24}$ and of a master symmetry ${ }^{35}$ from which one is able to construct an infinite hierarchy of generalized symmetries. The problem of finding the Bäcklund transformation and Lax pair in the general case when all the parameters are different from zero seems to be still open. Some partial results are contained in Ref. 4.

In Refs. 25 and 26, the authors constructed a set of five conditions necessary for the existence of generalized symmetries for (1). They used the conditions to propose the integrability of a few

\footnotetext{
a)E-mail: gubbiotti@mat.uniroma3.it

b) E-mail: scimiterna@ fis.uniroma3.it

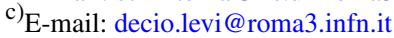


equations: a non-autonomous generalization of the Toda lattice, of the Volterra equation, and of YdKN. These are the only key equations which, up to Miura transformations, appear in the classification of differential difference equations on three points. Here, in the following, for its relevance in the present work, we present just the non-autonomous generalization of the YdKN

$$
\frac{\mathrm{d} q_{k}}{\mathrm{~d} t}=\frac{A_{k}\left(q_{k}\right) q_{k+1} q_{k-1}+B_{k}\left(q_{k}\right)\left(q_{k+1}+q_{k-1}\right)+C_{k}\left(q_{k}\right)}{q_{k+1}-q_{k-1}},
$$

where the $k$-dependent coefficients are now given by

$$
\begin{gathered}
A_{k}\left(q_{k}\right)=a q_{k}^{2}+2 b_{k} q_{k}+c_{k}, \\
B_{k}\left(q_{k}\right)=b_{k+1} q_{k}^{2}+d q_{k}+e_{k+1}, \\
C_{k}\left(q_{k}\right)=c_{k+1} q_{k}^{2}+2 e_{k} q_{k}+f
\end{gathered}
$$

with $b_{k}, c_{k}$, and $e_{k}$ 2-periodic functions. Eq. (4) has conservation laws of second and third order and two generalized local symmetries of order $i$ and $i+1$, with $i<4$.

It was proved in Ref. 23 that the three-point symmetries of the equations belonging to the socalled ABS classification, ${ }^{1}$ found systematically in Ref. 28 , are all particular cases of (2). Here in this note, we will show that the three-point generalized symmetries of all the equations coming from the classification of Boll, ${ }^{2,6-8}$ which extends the ABS one, ${ }^{1}$ are all particular cases of the YdKN or the non-autonomous YdKN. In particular, we will present the symmetries of all the classes of equations $H^{4}$ and $H^{6}$, noting that the symmetries of the rhombic $H^{4}$ have been found first in Ref. 32. For the remaining classes of equations, namely, the trapezoidal $H^{4}$ and the $H^{6}$ equations, which were found to be linearizable in Ref. 19, this is the first time that their generalized symmetries are presented. Furthermore, we also present a new suggestion for the integrability of the non-autonomous YdKN (4) based on the algebraic entropy test and use the same criterion to prove the integrability of the other non-autonomous equations of the $H^{4}$ and $H^{6}$ classes.

In Section II, we present the three-point generalized symmetries of the $H^{4}$ and $H^{6}$ classes and identify them with subcases of the YdKN or of its non-autonomous extension. We present in Section III the master symmetries associated with $H^{4}$ and $H^{6}$ classes, while in Section IV, we compute the algebraic entropy for the non-autonomous YdKN and its subcases obtained before. In Section V, we present some brief conclusions.

\section{THREE POINT GENERALIZED SYMMETRIES AND THEIR IDENTIFICATION}

In this section, we consider the various classes of equations coming from the classification of Boll, ${ }^{2,6-8}$ as presented in Ref. 19, show their symmetries and show the identification of the fluxes of such symmetries with the YdKN and its non-autonomous extension.

\section{A. Rhombic $H^{4}$ equations}

Once written on the $\mathbb{Z}_{(n, m)}^{2}$ lattice, according to Ref. 32, the three equations belonging to this class have the form

$$
\begin{aligned}
{ }_{r} H_{1}^{\varepsilon} & :\left(u_{n, m}-u_{n+1, m+1}\right)\left(u_{n+1, m}-u_{n, m+1}\right)-(\alpha-\beta) \\
& +\varepsilon(\alpha-\beta)\left(F_{n+m}^{(+)} u_{n+1, m} u_{n, m+1}+F_{n+m}^{(-)} u_{n, m} u_{n+1, m+1}\right)=0, \\
{ }_{r} H_{2}^{\varepsilon} & :\left(u_{n, m}-u_{n+1, m+1}\right)\left(u_{n+1, m}-u_{n, m+1}\right)+ \\
& +(\beta-\alpha)\left(u_{n, m}+u_{n+1, m}+u_{n, m+1}+u_{n+1, m+1}\right)-\alpha^{2}+\beta^{2} \\
& -\varepsilon(\beta-\alpha)^{3}-\varepsilon(\beta-\alpha)\left(2 F_{n+m}^{(-)} u_{n, m}+2 F_{n+m}^{(+)} u_{n+1, m}+\alpha+\beta\right) . \\
& \cdot\left(2 F_{n+m}^{(-)} u_{n+1, m+1}+2 F_{n+m}^{(+)} u_{n, m+1}+\alpha+\beta\right)=0,
\end{aligned}
$$




$$
\begin{aligned}
{ }_{r} H_{3}^{\varepsilon} & : \alpha\left(u_{n, m} u_{n+1, m}+u_{n, m+1} u_{n+1, m+1}\right) \\
& -\beta\left(u_{n, m} u_{n, m+1}+u_{n+1, m} u_{n+1, m+1}\right)+\left(\alpha^{2}-\beta^{2}\right) \delta \\
& -\frac{\varepsilon\left(\alpha^{2}-\beta^{2}\right)}{\alpha \beta}\left(F_{n+m}^{(+)} u_{n+1, m} u_{n, m+1}+F_{n+m}^{(-)} u_{n, m} u_{n+1, m+1}\right)=0,
\end{aligned}
$$

where

$$
F_{k}^{ \pm}=\frac{1 \pm(-1)^{k}}{2}, \quad k \in \mathbb{Z} .
$$

Their three-point symmetries ${ }^{32}$ are given by

$$
\begin{aligned}
& \widehat{X}_{n}^{r} H_{1}^{\varepsilon}=\frac{1-\varepsilon\left(F_{n+m}^{(+)} u_{n+1, m} u_{n-1, m}+F_{n+m}^{(-)} u_{n, m}^{2}\right)}{u_{n+1, m}-u_{n-1, m}} \partial_{u_{n, m}}, \\
& \widehat{X}_{m}^{r} H_{1}^{\varepsilon}=\frac{1-\varepsilon\left(F_{n+m}^{(+)} u_{n, m+1} u_{n, m-1}+F_{n+m}^{(-)} u_{n, m}^{2}\right)}{u_{n, m+1}-u_{n, m-1}} \partial_{u_{n, m}}, \\
& \widehat{X}_{n}^{r} H_{2}^{\varepsilon}=\left[\frac{\left(1-4 \varepsilon \alpha F_{n+m}^{(-)}\right)\left(u_{n+1, m}+u_{n-1, m}\right)-4 \varepsilon F_{n+m}^{(+)} u_{n+1, m} u_{n-1, m}}{u_{n+1, m}-u_{n-1, m}}+\right. \\
& \left.+\frac{2 \alpha-4 \varepsilon \alpha^{2}-4 \varepsilon F_{n+m}^{(-)} u_{n, m}^{2}+\left(1-4 \varepsilon \alpha F_{n+m}^{(-)}\right) u_{n, m}}{u_{n+1, m}-u_{n-1, m}}\right] \partial_{u_{n, m}}, \\
& \widehat{X}_{m}^{r} H_{2}^{\varepsilon}=\left[\frac{\left(1-4 \varepsilon \beta F_{n+m}^{(-)}\right)\left(u_{n, m+1}+u_{n, m-1}\right)-4 \varepsilon F_{n+m}^{(+)} u_{n, m+1} u_{n, m-1}}{u_{n, m+1}-u_{n, m-1}}+\right. \\
& \left.+\frac{2 \beta-4 \varepsilon \beta^{2}-4 \varepsilon F_{n+m}^{(-)} u_{n, m}^{2}+\left(1-4 \varepsilon \beta F_{n+m}^{(-)}\right) u_{n, m}}{u_{n, m+1}+u_{n, m-1}}\right] \partial_{u_{n, m}}, \\
& \widehat{X}_{n}^{r} H_{3}^{\varepsilon}=\left[\frac{1}{2} \frac{u_{n, m}\left(u_{n+1, m}+u_{n-1, m}\right)+2 \delta \alpha}{u_{n+1, m}-u_{n-1, m}}-\right. \\
& \left.-\frac{\varepsilon}{\alpha} \frac{\left(F_{n+m}^{(+)} u_{n+1, m} u_{n-1, m}+F_{n+m}^{(-)} u_{n, m}^{2}\right)}{u_{n+1, m}-u_{n-1, m}}\right] \partial_{u_{n, m}}, \\
& \widehat{X}_{m}^{r} H_{3}^{\varepsilon}=\left[\frac{1}{2} \frac{u_{n, m}\left(u_{n, m+1}+u_{n, m-1}\right)+2 \delta \beta}{u_{n, m+1}+u_{n, m-1}}-\right. \\
& \left.-\frac{\varepsilon}{\beta} \frac{\left(F_{n+m}^{(+)} u_{n, m+1} u_{n, m-1}+F_{n+m}^{(-)} u_{n, m}^{2}\right)}{u_{n, m+1}+u_{n, m-1}}\right] \partial_{u_{n, m}} .
\end{aligned}
$$

As stated in Ref. 32, the fluxes of the symmetries (8) are readily identified with the corresponding cases of the non-autonomous YdKN Equation (4). Such identification is, in this paper, made explicit by showing the appropriate values of the coefficients of (4) in Table I.

\section{B. Trapezoidal $\boldsymbol{H}^{4}$ equations}

We now consider the trapezoidal $H^{4}$ equations, which appeared in Refs. 7 and 8 and whose non-autonomous form was given in Ref. 19

$$
\begin{aligned}
{ }_{t} H_{1}: & \left(u_{n, m}-u_{n+1, m}\right)\left(u_{n, m+1}-u_{n+1, m+1}\right)- \\
& -\alpha_{2} \varepsilon^{2}\left(F_{m}^{(+)} u_{n, m+1} u_{n+1, m+1}+F_{m}^{(-)} u_{n, m} u_{n+1, m}\right)-\alpha_{2}=0,
\end{aligned}
$$


TABLE I. Identification of the coefficients in the symmetries of the rhombic $H^{4}$ equations with those of the non-autonomous YdKN equation.

\begin{tabular}{|c|c|c|c|c|c|c|c|}
\hline Eq. & $k$ & $a$ & $b_{k}$ & $c_{k}$ & $d$ & $e_{k}$ & $f$ \\
\hline \multirow[t]{2}{*}{${ }_{r} H_{1}^{\varepsilon}$} & $n$ & 0 & 0 & $-\varepsilon F_{n+m}^{(+)}$ & 0 & 0 & 1 \\
\hline & $m$ & 0 & 0 & $-\varepsilon F_{n+m}^{(+)}$ & 0 & 0 & 1 \\
\hline \multirow[t]{2}{*}{${ }_{r} H_{2}^{\varepsilon}$} & $n$ & 0 & 0 & $-4 \varepsilon F_{n+m}^{(+)}$ & 0 & $1-4 \varepsilon \alpha F_{n+m}^{(-)}$ & $2 \alpha-4 \varepsilon \alpha^{2}$ \\
\hline & $m$ & 0 & 0 & $-4 \varepsilon F_{n+m}^{(+)}$ & 0 & $1-4 \varepsilon \beta F_{n+m}^{(-)}$ & $2 \beta-4 \varepsilon \beta^{2}$ \\
\hline \multirow[t]{2}{*}{$r H_{3}^{\varepsilon}$} & $n$ & 0 & 0 & $-\frac{\varepsilon F_{n+m}^{(+)}}{\alpha}$ & $\frac{1}{2}$ & 0 & $\delta \alpha$ \\
\hline & $m$ & 0 & 0 & $-\frac{\varepsilon F_{n+m}^{(+)}}{\beta}$ & $\frac{1}{2}$ & 0 & $\delta \beta$ \\
\hline
\end{tabular}

$$
\begin{aligned}
{ }_{t} H_{2}: & \left(u_{n, m}-u_{n+1, m}\right)\left(u_{n, m+1}-u_{n+1, m+1}\right) \\
& -\alpha_{2}\left(u_{n, m}+u_{n+1, m}+u_{n, m+1}+u_{n+1, m+1}\right) \\
& +\frac{\varepsilon \alpha_{2}}{2}\left(2 F_{m}^{(+)} u_{n, m+1}+2 \alpha_{3}+\alpha_{2}\right)\left(2 F_{m}^{(+)} u_{n+1, m+1}+2 \alpha_{3}+\alpha_{2}\right) \\
& +\frac{\varepsilon \alpha_{2}}{2}\left(2 F_{m}^{(-)} u_{n, m}+2 \alpha_{3}+\alpha_{2}\right)\left(2 F_{m}^{(-)} u_{n+1, m}+2 \alpha_{3}+\alpha_{2}\right) \\
& +\left(\alpha_{3}+\alpha_{2}\right)^{2}-\alpha_{3}^{2}-2 \varepsilon \alpha_{2} \alpha_{3}\left(\alpha_{3}+\alpha_{2}\right)=0, \\
{ }_{t} H_{3}: & \alpha_{2}\left(u_{n, m} u_{n+1, m+1}+u_{n+1, m} u_{n, m+1}\right) \\
& -\left(u_{n, m} u_{n, m+1}+u_{n+1, m} u_{n+1, m+1}\right)-\alpha_{3}\left(\alpha_{2}^{2}-1\right) \delta^{2}+ \\
& \quad-\frac{\varepsilon^{2}\left(\alpha_{2}^{2}-1\right)}{\alpha_{3} \alpha_{2}}\left(F_{m}^{(+)} u_{n, m+1} u_{n+1, m+1}+F_{m}^{(-)} u_{n, m} u_{n+1, m}\right)=0 .
\end{aligned}
$$

We can easily calculate the three-point symmetries of ${ }_{t} H_{2}^{\varepsilon}(9 \mathrm{~b})$ and of ${ }_{t} H_{3}^{\varepsilon}(9 \mathrm{c})$,

$$
\begin{aligned}
\widehat{X}_{n}^{H_{2}^{\varepsilon}}= & {\left[\frac{\left(u_{n, m}+\varepsilon \alpha_{2}^{2} F_{m}^{(+)}\right)\left(u_{n+1, m}+u_{n-1, m}\right)-u_{n+1, m} u_{n-1, m}}{u_{n+1, m}-u_{n-1, m}}-\right.} \\
& \left.-\frac{u_{n, m}^{2}-2 \varepsilon F_{m}^{(+)} \alpha_{2}^{2} u_{n, m}-\alpha_{2}^{2}+4 \varepsilon F_{m}^{(+)} \alpha_{2}^{3}+8 \varepsilon F_{m}^{(+)} \alpha_{2}^{2} \alpha_{3}+\varepsilon^{2} F_{m}^{(+)} \alpha_{2}^{4}}{u_{n+1, m}-u_{n-1, m}}\right] \partial_{u_{n, m}}, \\
\widehat{X}_{m}^{t} H_{2}^{\varepsilon} & =\left[\frac{\left[\frac{1}{2}-\varepsilon\left(\alpha_{2}+\alpha_{3}\right) F_{m}^{(+)}\right]\left(u_{n, m+1}+u_{n, m-1}\right)-\varepsilon F_{m}^{(+)} u_{n, m+1} u_{n, m-1}}{u_{n, m+1}-u_{n, m-1}}-\right. \\
& \left.-\frac{\varepsilon F_{m}^{(-)} u_{n, m}^{2}-\left[1-2 \varepsilon\left(\alpha_{2}+\alpha_{3}\right) F_{m}^{(-)}\right] u_{n, m}+\alpha_{3}+\varepsilon\left(\alpha_{2}+\alpha_{3}\right)^{2}}{u_{n, m+1}-u_{n, m-1}}\right] \partial_{u_{n, m}}, \\
\widehat{X}_{n}^{t} H_{3}^{\varepsilon} & =\left[\frac{\frac{1}{2} \alpha_{2}\left(1+\alpha_{2}^{2}\right) u_{n, m}\left(u_{n+1, m}+u_{n-1, m}\right)-\alpha_{2}^{2} u_{n+1, m} u_{n-1, m}}{u_{n+1, m}-u_{n-1, m}}-\right. \\
& \left.-\frac{\alpha_{2}^{2} u_{n, m}^{2}+\varepsilon^{2} \delta^{2}\left(1-\alpha_{2}^{2}\right)^{2} F_{m}^{(+)}}{u_{n+1, m}-u_{n-1, m}}\right] \partial_{u_{n, m}}, \\
\widehat{X}_{m}^{t} H_{3}^{\varepsilon}= & {\left[\frac{\frac{1}{2} \alpha_{3} u_{n, m}\left(u_{n, m+1}+u_{n, m-1}\right)-\varepsilon^{2} F_{m}^{(+)} u_{n, m+1} u_{n, m-1}}{u_{n, m+1}-u_{n, m-1}}-\right.} \\
& \left.-\frac{\varepsilon^{2} F_{m}^{(-)} u_{n, m}^{2}+\alpha_{3}^{2} \delta^{2}}{u_{n, m+1}-u_{n, m-1}}\right] \partial_{u_{n, m}} .
\end{aligned}
$$


The symmetries in the $n$ and $m$ directions and the linearizations of the ${ }_{t} H_{1}^{\varepsilon}$ Equation (9a) have been presented in Ref. 20. Their peculiarity is that they are written in terms of two arbitrary functions of one continuous variable and one discrete index and by arbitrary functions of the lattice variables. This is the first time that we find a lattice equation whose generalized symmetries depend on arbitrary functions. Almost surely, this peculiarity is related to the very specific way in which ${ }_{t} H_{1}^{\varepsilon}$ is linearizable. ${ }^{18}$ Here we present only the sub-cases which are related to the YdKN equation in its autonomous or non-autonomous form.

The general symmetry in the $n$ direction is

$$
\begin{aligned}
\hat{X}_{n}^{t} H_{1}^{\varepsilon}= & F_{m}^{(+)}\left\{\frac{\alpha_{2}\left(v^{2}+\varepsilon^{2} \alpha_{2}^{2}\right)}{(r-v)(r+v)} B_{n}\left(\frac{\alpha_{2}}{r}\right)-\frac{\alpha_{2}\left(r^{2}+\varepsilon^{2} \alpha_{2}^{2}\right)}{(r-v)(r+v)} B_{n-1}\left(\frac{\alpha_{2}}{v}\right)+\right. \\
& \left.+\left[u_{n, m}-\frac{\left(r^{2}+\varepsilon^{2} \alpha_{2}^{2}\right) v}{(r-v)(r+v)}\right] \alpha+\gamma_{m}\right\} \partial_{u_{n, m}}+F_{m}^{(-)}\left[\frac { s ^ { 2 } t ^ { 2 } } { ( s - t ) ( s + t ) } \left(B_{n}(s)-\right.\right. \\
& \left.\left.-B_{n-1}(t)\right)-\frac{s^{2} t}{(s-t)(s+t)} \alpha+\delta_{m}\right]\left(1+\varepsilon^{2} u_{n, m}^{2}\right) \partial_{u_{n, m}}, \quad r \doteq u_{n+1, m}-u_{n, m}, \\
s \doteq & \frac{u_{n+1, m}-u_{n, m}}{1+\varepsilon^{2} u_{n+1, m} u_{n, m}}, \quad t \doteq \frac{u_{n, m}-u_{n-1, m}}{1+\varepsilon^{2} u_{n-1, m} u_{n, m}}, \quad v \doteq u_{n, m}-x_{u-1, m},
\end{aligned}
$$

where $B_{n}(x), \gamma_{m}$, and $\delta_{m}$ are generic functions of their arguments and $\alpha$ is an arbitrary parameter. When $B_{n}(x)=-1 / x, \alpha=\gamma_{m}=\delta_{m}=0$, we get a symmetry of YdKN type

$$
\hat{X}_{n}^{t H_{1}^{\varepsilon}}=\left[\frac{\left(u_{n+1, m}-u_{n, m}\right)\left(u_{n, m}-u_{n-1, m}\right)}{u_{n+1, m}-u_{n-1, m}}-F_{m}^{(+)} \frac{\varepsilon^{2} \alpha_{2}^{2}}{u_{n+1, m}-u_{n-1, m}}\right] \partial_{u_{n, m}} .
$$

The general symmetry in the $m$ direction is

$$
\begin{aligned}
\widehat{X}_{m}^{H_{1}^{\varepsilon}}= & {\left[F_{m}^{(+)}\left(B_{m}\left(\frac{u_{n, m+1}-u_{n, m-1}}{1+\varepsilon^{2} u_{n, m+1} u_{n, m-1}}\right)+\kappa_{m}\right)\right.} \\
& \left.+F_{m}^{(-)}\left(1+\varepsilon^{2} u_{n, m}^{2}\right)\left(C_{m}\left(u_{n, m+1}-u_{n, m-1}\right)+\lambda_{m}\right)\right] \partial_{u_{n, m}} .
\end{aligned}
$$

When $B_{m}(t)=1 / t, C_{m}(t)=1 / t$ and $\kappa_{m}=\lambda_{m}=0,(13)$ becomes

$$
\widehat{X}_{m}^{t} H_{1}^{\varepsilon}=\left[F_{m}^{(+)} \frac{1+\varepsilon^{2} u_{n, m+1} u_{n, m-1}}{u_{n, m+1}-u_{n, m-1}}+F_{m}^{(-)} \frac{1+\varepsilon^{2} u_{n, m}^{2}}{u_{n, m+1}-u_{n, m-1}}\right] \partial_{u_{n, m}},
$$

a symmetry of YdKN type.

In both cases, (11) and (13), we also searched for other integrable subcases by looking for those subcases of the functions $B_{m}, C_{m}$, and $B_{n}$ such that (11) and (13) reduce either to the non-autonomous Toda lattice or to the non-autonomous Volterra equation. The result was null. In this calculation, we considered arbitrary expressions for $B_{n}, B_{m}$, and $C_{m}$. Still there might be some other integrable choice. The proof that there is none would require the application of the integrability conditions, as introduced in Ref. 35, to (11) and (13). However the expressions are too complicate and we have not been able to deal with them. Moreover (12) and (14) are the most general equations of YdKN type contained in (11) and (13).

Let us notice that the symmetries (10) and (12) in the $n$ direction are sub-cases of the original YdKN equation. As $F_{m}^{( \pm)}$depends on the other lattice index, it can be treated like a parameter which is either 0 or 1 .

The explicit identification of the coefficients of the symmetries (10), (12), and (14) is shown in Table II. 
TABLE II. Identification of the coefficients in the symmetries of the trapezoidal $H^{4}$ equations with those of the YdKN equation. In the direction $n$, the $\mathrm{YdKN}$ is autonomous while in the $m$ direction is non-autonomous. Here the symmetries of ${ }_{t} H_{1}^{\varepsilon}$ in the $m$ direction are the subcase (14) of (13) while those in the $n$ direction are the subcase (12) of (11).

\begin{tabular}{cccccccc}
\hline \hline Eq. & $k$ & $a$ & $b_{k}$ & $c_{k}$ & $d$ & $e_{k}$ & $f$ \\
\hline${ }_{t} H_{1}^{\varepsilon}$ & $n$ & 0 & 0 & -1 & 1 & 0 & $-\varepsilon^{2} \alpha_{2}^{2} F_{m}^{(+)}$ \\
& $m$ & 0 & 0 & $\varepsilon^{2} F_{m}^{(+)}$ & 0 & 0 & 2 \\
${ }_{t} H_{2}^{\varepsilon}$ & $n$ & 0 & 0 & -1 & 1 & $\varepsilon \alpha_{2}^{2} F_{m}^{(+)}$ & $\alpha_{2}^{2}-\varepsilon \alpha_{2}^{2}\left(4 \alpha_{2}+8 \alpha_{3}+\varepsilon \alpha_{2}^{2}\right) F_{m}^{(+)}$ \\
& $m$ & 0 & 0 & $-\varepsilon F_{m}^{(+)}$ & 0 & $\frac{1}{2}-\varepsilon\left(\alpha_{2}+\alpha_{3}\right) F_{m}^{(+)}$ & $-\alpha_{3}-\varepsilon\left(\alpha_{2}+\alpha_{3}\right)^{2}$ \\
${ }_{t} H_{3}^{\varepsilon}$ & $n$ & 0 & 0 & $-\alpha_{2}^{2}$ & $\frac{1}{2} \alpha_{2}\left(1+\alpha_{2}^{2}\right)$ & 0 & $-\varepsilon^{2} \delta^{2} F_{m}^{(+)}\left(1-\alpha_{2}^{2}\right)^{2}$ \\
& $m$ & 0 & 0 & $-\varepsilon^{2} F_{m}^{(+)}$ & $\frac{1}{2} \alpha_{3}$ & 0 & $-\alpha_{3}^{2} \delta^{2}$ \\
\hline \hline
\end{tabular}

\section{C. $H^{6}$ equations}

In this subsection, we consider the equations of the family $H^{6}$ introduced in Refs. 7 and 8. We shall present their non-autonomous form on the lattice $\mathbb{Z}_{(n, m)}^{2}$ as given in Ref. 19

$$
\begin{aligned}
& { }_{1} D_{2}:\left(F_{n+m}^{(-)}-\delta_{1} F_{n}^{(+)} F_{m}^{(-)}+\delta_{2} F_{n}^{(+)} F_{m}^{(+)}\right) u_{n, m} \\
& +\left(F_{n+m}^{(+)}-\delta_{1} F_{n}^{(-)} F_{m}^{(-)}+\delta_{2} F_{n}^{(-)} F_{m}^{(+)}\right) u_{n+1, m}+ \\
& +\left(F_{n+m}^{(+)}-\delta_{1} F_{n}^{(+)} F_{m}^{(+)}+\delta_{2} F_{n}^{(+)} F_{m}^{(-)}\right) u_{n, m+1} \\
& +\left(F_{n+m}^{(-)}-\delta_{1} F_{n}^{(-)} F_{m}^{(+)}+\delta_{2} F_{n}^{(-)} F_{m}^{(-)}\right) u_{n+1, m+1}+ \\
& +\delta_{1}\left(F_{m}^{(-)} u_{n, m} u_{n+1, m}+F_{m}^{(+)} u_{n, m+1} u_{n+1, m+1}\right) \\
& +F_{n+m}^{(+)} u_{n, m} u_{n+1, m+1}+F_{n+m}^{(-)} u_{n+1, m} u_{n, m+1}=0 \text {, } \\
& { }_{2} D_{2}:\left(F_{m}^{(-)}-\delta_{1} F_{n}^{(+)} F_{m}^{(-)}+\delta_{2} F_{n}^{(+)} F_{m}^{(+)}-\delta_{1} \lambda F_{n}^{(-)} F_{m}^{(+)}\right) u_{n, m} \\
& +\left(F_{m}^{(-)}-\delta_{1} F_{n}^{(-)} F_{m}^{(-)}+\delta_{2} F_{n}^{(-)} F_{m}^{(+)}-\delta_{1} \lambda F_{n}^{(+)} F_{m}^{(+)}\right) u_{n+1, m} \\
& +\left(F_{m}^{(+)}-\delta_{1} F_{n}^{(+)} F_{m}^{(+)}+\delta_{2} F_{n}^{(+)} F_{m}^{(-)}-\delta_{1} \lambda F_{n}^{(-)} F_{m}^{(-)}\right) u_{n, m+1} \\
& +\left(F_{m}^{(+)}-\delta_{1} F_{n}^{(-)} F_{m}^{(+)}+\delta_{2} F_{n}^{(-)} F_{m}^{(-)}-\delta_{1} \lambda F_{n}^{(+)} F_{m}^{(-)}\right) u_{n+1, m+1} \\
& +\delta_{1}\left(F_{n+m}^{(-)} u_{n, m} u_{n+1, m+1}+F_{n+m}^{(+)} u_{n+1, m} u_{n, m+1}\right) \\
& +F_{m}^{(+)} u_{n, m} u_{n+1, m}+F_{m}^{(-)} u_{n, m+1} u_{n+1, m+1}-\delta_{1} \delta_{2} \lambda=0, \\
& { }_{3} D_{2}:\left(F_{m}^{(-)}-\delta_{1} F_{n}^{(-)} F_{m}^{(-)}+\delta_{2} F_{n}^{(+)} F_{m}^{(+)}-\delta_{1} \lambda F_{n}^{(-)} F_{m}^{(+)}\right) u_{n, m} \\
& +\left(F_{m}^{(-)}-\delta_{1} F_{n}^{(+)} F_{m}^{(-)}+\delta_{2} F_{n}^{(-)} F_{m}^{(+)}-\delta_{1} \lambda F_{n}^{(+)} F_{m}^{(+)}\right) u_{n+1, m} \\
& +\left(F_{m}^{(+)}-\delta_{1} F_{n}^{(-)} F_{m}^{(+)}+\delta_{2} F_{n}^{(+)} F_{m}^{(-)}-\delta_{1} \lambda F_{n}^{(-)} F_{m}^{(-)}\right) u_{n, m+1} \\
& +\left(F_{m}^{(+)}-\delta_{1} F_{n}^{(+)} F_{m}^{(+)}+\delta_{2} F_{n}^{(-)} F_{m}^{(-)}-\delta_{1} \lambda F_{n}^{(+)} F_{m}^{(-)}\right) u_{n+1, m+1} \\
& +\delta_{1}\left(F_{n}^{(-)} u_{n, m} u_{n, m+1}+F_{n}^{(+)} u_{n+1, m} u_{n+1, m+1}\right) \\
& +F_{m}^{(-)} u_{n, m+1} u_{n+1, m+1}+F_{m}^{(+)} u_{n, m} u_{n+1, m}-\delta_{1} \delta_{2} \lambda=0 \text {, } \\
& D_{3}: F_{n}^{(+)} F_{m}^{(+)} u_{n, m}+F_{n}^{(-)} F_{m}^{(+)} u_{n+1, m}+F_{n}^{(+)} F_{m}^{(-)} u_{n, m+1} \\
& +F_{n}^{(-)} F_{m}^{(-)} u_{n+1, m+1}+F_{m}^{(-)} u_{n, m} u_{n+1, m} \\
& +F_{n}^{(-)} u_{n, m} u_{n, m+1}+F_{n+m}^{(-)} u_{n, m} u_{n+1, m+1}+ \\
& +F_{n+m}^{(+)} u_{n+1, m} u_{n, m+1}+F_{n}^{(+)} u_{n+1, m} u_{n+1, m+1} \\
& +F_{m}^{(+)} u_{n, m+1} u_{n+1, m+1}=0 \text {, }
\end{aligned}
$$




$$
\begin{aligned}
{ }_{1} D_{4} & : \delta_{1}\left(F_{n}^{(-)} u_{n, m} u_{n, m+1}+F_{n}^{(+)} u_{n+1, m} u_{n+1, m+1}\right)+ \\
& +\delta_{2}\left(F_{m}^{(-)} u_{n, m} u_{n+1, m}+F_{m}^{(+)} u_{n, m+1} u_{n+1, m+1}\right)+ \\
& +u_{n, m} u_{n+1, m+1}+u_{n+1, m} u_{n, m+1}+\delta_{3}=0, \\
{ }_{2} D_{4}: & \delta_{1}\left(F_{n}^{(-)} u_{n, m} u_{n, m+1}+F_{n}^{(+)} u_{n+1, m} u_{n+1, m+1}\right)+ \\
& +\delta_{2}\left(F_{n+m}^{(-)} u_{n, m} u_{n+1, m+1}+F_{n+m}^{(+)} u_{n+1, m} u_{n, m+1}\right)+ \\
& +u_{n, m} u_{n+1, m}+u_{n, m+1} u_{n+1, m+1}+\delta_{3}=0 .
\end{aligned}
$$

The three forms of the equation $D_{2},(15 \mathrm{a}),(15 \mathrm{~b})$, and (15c), which we will collectively call ${ }_{i} D_{2}$ assuming $i$ in $\{1,2,3\}$, possess symmetries in the $n$ and in the $m$ direction. The symmetries $\widehat{Z}_{j}^{i D_{2}}$ with $i$ in $\{1,2,3\}$ and $j=(m, n)$ are a linear combination of one point and three-point symmetries

$$
\widehat{Z}_{j}^{i D_{2}}=\widehat{X}_{j}^{i D_{2}}+K_{1} \widehat{Y}_{1}^{i D_{2}}+K_{2} \widehat{Y}_{2}^{i D_{2}}, \quad j=n, m, i=1,2,3 .
$$

For convenience of presentation, we write them down separately

$$
\begin{aligned}
& \widehat{X}_{n}^{1 D_{2}}=\left[\frac{\left(F_{n}^{(+)} F_{m}^{(-)}-\delta_{1} F_{n}^{(+)} F_{m}^{(+)}\right)\left(u_{n, m+1}+u_{n-1, m}\right)}{u_{n+1, m}-u_{n-1, m}}+\right. \\
& +\frac{\left(F_{n}^{(+)} F_{m}^{(-)}-\delta_{1} F_{n}^{(+)} F_{m}^{(+)}-\delta_{1} \delta_{2}\right) F_{n}^{(-)} F_{m}^{(+)} u_{n-1, m}}{u_{n+1, m}-u_{n-1, m}}+ \\
& \left.+\frac{\left(F_{n+m}^{(+)}-\delta_{1} F_{m}^{(+)}-\delta_{1} \delta_{2} F_{n}^{(+)} F_{m}^{(+)}\right) u_{n, m}+\delta_{2} F_{m}^{(-)}}{u_{n+1, m}-u_{n-1, m}}\right] \partial_{u_{n, m}}, \\
& \widehat{X}_{m}^{1} D_{2}=\left[\frac{\delta_{1} F_{n}^{(-)} F_{m}^{(+)} u_{n, m+1} u_{n, m-1}+F_{n+m}^{(-)}\left(u_{n, m+1}+u_{n, m-1}\right)}{u_{n, m+1}-u_{n, m-1}}+\right. \\
& +\frac{\delta_{1} F_{m}^{(+)} u_{n, m+1}+\delta_{1} \delta_{2} F_{n}^{(-)} F_{m}^{(+)} u_{n, m-1}+\delta_{1} F_{n}^{(-)} F_{m}^{(-)} u_{n, m}^{2}}{u_{n, m+1}-u_{n, m-1}}+ \\
& +\frac{\left[F_{n+m}^{(+)}+\delta_{1}\left(F_{n}^{(+)} F_{m}^{(-)}-F_{n}^{(-)} F_{m}^{(-)}\right)+\delta_{1} \delta_{2} F_{n}^{(-)} F_{m}^{(-)}\right] u_{n, m}}{u_{n, m+1}-u_{n, m-1}}- \\
& \left.-\frac{\delta_{2}\left(\delta_{1}-1\right) F_{n}^{(-)}}{u_{n, m+1}-u_{n, m-1}}\right] \partial_{u n m} \\
& \widehat{X}_{n}^{2 D_{2}}=\left[\frac{\left(F_{n}^{(-)} F_{m}^{(-)} \delta_{1}+F_{n}^{(-)} F_{m}^{(-)} \delta_{1} \delta_{2}-F_{n}^{(-)} F_{m}^{(-)}\right) u_{n+1, m}+\left(F_{n}^{(+)} F_{m}^{(+)} \delta_{1}-F_{n}^{(+)} F_{m}^{(-)}\right) u_{n-1, m}}{u_{n+1, m}-u_{n-1, m}}+\right. \\
& \left.+\frac{\left(\delta_{1} F_{n+m}^{(-)}-F_{m}^{(-)}+\delta_{1} \delta_{2} F_{n}^{(+)} F_{m}^{(-)}\right) u_{n, m}-\left(\delta_{1}-1\right) F_{m}^{(+)}}{u_{n+1, m}-u_{n-1, m}}\right] \partial_{u_{n, m}}, \\
& \widehat{X}_{m}^{2 D_{2}}=\left[\frac{F_{n}^{(-)} F_{m}^{(-)} \delta_{1} u_{n, m+1} u_{n, m-1}+\left(\delta_{1} \delta_{2} F_{n}^{(-)} F_{m}^{(-)}+F_{n}^{(+)} F_{m}^{(-)}\right) u_{n, m+1}}{u_{n, m+1}-u_{n, m-1}}+\right. \\
& +\frac{+\left(\delta_{1} F_{n}^{(+)} F_{m}^{(+)}+F_{n}^{(-)} F_{m}^{(-)}-\delta_{1} F_{n}^{(-)} F_{m}^{(-)}\right) u_{n, m-1}}{u_{n, m+1}-u_{n, m-1}}+ \\
& +\frac{\delta_{1} F_{n}^{(-)} F_{m}^{(+)} u_{n, m}^{2}+\left[F_{n}^{(+)} F_{m}^{(-)}+\left(\delta_{2}-1\right) F_{n}^{(-)} F_{m}^{(+)}+F_{m}^{(+)}\right] u_{n, m}}{u_{n, m+1}-u_{n, m-1}}+ \\
& \left.+\frac{\delta_{2}\left(1-\delta_{2}\right) F_{n}^{(-)}-\delta_{1} \lambda F_{n}^{(+)}}{u_{n, m+1}-u_{n, m-1}}\right] \partial_{u_{n, m}},
\end{aligned}
$$




$$
\begin{aligned}
& \widehat{X}_{n}^{3} D_{2}=\left[\frac{\left(\delta_{1} F_{n}^{(+)} F_{m}^{(-)}+\delta_{1} \delta_{2} F_{n}^{(+)} F_{m}^{(-)}-F_{n}^{(+)} F_{m}^{(-)}\right) u_{n+1, m}}{u_{n+1, m}-u_{n-1, m}}+\right. \\
& +\frac{\left(F_{n}^{(+)} F_{m}^{(+)} \delta_{1}-F_{n}^{(-)} F_{m}^{(-)}\right) u_{n-1, m}}{u_{n+1, m}-u_{n-1, m}}+ \\
& \left.+\frac{\left(\delta_{1} F_{n}^{(-)} F_{m}^{(-)} \delta_{2}+F_{n}^{(-)} \delta_{1}-F_{m}^{(-)}\right) u_{n, m}+\left(1-\delta_{1}\right) F_{m}^{(+)}}{u_{n+1, m}-u_{n-1, m}}\right] \partial u_{n, m}, \\
& \widehat{X}_{m}^{3} D_{2}=\left[\frac{\left(1-\delta_{1}-\delta_{1} \delta_{2}\right) F_{n}^{(+)} F_{m}^{(-)} u_{n, m+1}}{u_{n, m+1}-u_{n, m-1}}+\right. \\
& +\frac{\left(F_{n}^{(-)} F_{m}^{(-)}-F_{n}^{(+)} F_{m}^{(+)} \delta_{1}\right) u_{n, m-1}+\delta_{2} F_{n}^{(-)}}{u_{n, m+1}-u_{n, m-1}}+ \\
& +\frac{\left(F_{m}^{(+)}-\delta_{1} F_{n}^{(+)}-\delta_{1} \delta_{2} F_{n}^{(+)} F_{m}^{(+)}\right) u_{n, m}}{u_{n, m+1}-u_{n, m-1}}- \\
& \left.-\frac{\lambda \delta_{1}\left(1-\delta_{1}-\delta_{1} \delta_{2}\right) F_{n}^{(+)}}{u_{n, m+1}-u_{n, m-1}}\right] \partial_{u n m} \text {, } \\
& \widehat{Y}_{1}^{1} D_{2}=\left(F_{n}^{(+)} F_{m}^{(+)}+F_{n}^{(+)} F_{m}^{(-)}+F_{n}^{(-)} F_{m}^{(+)}\right) u_{n, m} \partial_{u_{n, m}} \\
& \widehat{Y}_{2}^{1} D_{2}=\left[\delta_{1} F_{n}^{(+)} F_{m}^{(+)}+\left[1-\delta_{1}\left(1+\delta_{2}\right)\right] F_{n}^{(-)} F_{m}^{(+)}+F_{n}^{(+)} F_{m}^{(-)}\right] \partial_{u_{n, m}}, \\
& \widehat{Y}_{1}^{2 D_{2}}=\left[\left(F_{n}^{(+)} F_{m}^{(+)}+F_{n}^{(+)} F_{m}^{(-)}+F_{n}^{(-)} F_{m}^{(+)}\right) u_{n, m}-\right. \\
& \left.-\lambda F_{n}^{(+)} F_{m}^{(-)}+\lambda\left[1-\delta_{1}\left(1+\delta_{2}\right)\right] F_{n}^{(-)} F_{m}^{(-)}\right] \partial_{u_{n, m}} \text {, } \\
& \widehat{Y}_{2}^{2 D_{2}}=\left[\delta_{1} F_{n}^{(+)} F_{m}^{(+)}+F_{n}^{(+)} F_{m}^{(-)}\left[1-\delta_{1}\left(1+\delta_{2}\right)\right] F_{n}^{(-)} F_{m}^{(-)}\right] \partial_{u_{n, m}}, \\
& \widehat{Y}_{1}^{3} D_{2}=\left[\left(F_{n}^{(+)} F_{m}^{(+)}+F_{n}^{(+)} F_{m}^{(-)}+F_{n}^{(-)} F_{m}^{(+)}\right) u_{n, m}-\right. \\
& \left.-\lambda F_{n}^{(-)} F_{m}^{(-)}+\lambda\left[1-\delta_{1}\left(1+\delta_{2}\right)\right] F_{n}^{(-)} F_{m}^{(-)}\right] \partial_{u_{n, m}} \text {, } \\
& \widehat{Y}_{2}^{3 D_{2}}=\left[\delta_{1} F_{n}^{(+)} F_{m}^{(+)}+\left[1-\delta_{1}\left(1+\delta_{2}\right)\right] F_{n}^{(+)} F_{m}^{(-)}-F_{n}^{(-)} F_{m}^{(-)}\right] \partial_{u_{n, m}} .
\end{aligned}
$$

The identification of the resulting symmetries of equations ${ }_{i} D_{2}$ with the $\mathrm{YdKN}$ equation in the form (4) is displayed in Table III.

The $D_{3}$ Equation (15d) admits the symmetries $\widehat{Z}_{j}^{D_{3}}$ with $j=(m, n)$, where (note that equation $D_{3}$ (15d) is invariant under the exchange $n \leftrightarrow m$ so the symmetry $X_{m}^{D_{3}}$ (20b) can be obtained from the symmetry $X_{n}^{D_{3}}$ (20b) performing such exchange)

$$
\begin{gathered}
\widehat{Z}_{j}^{D_{3}}=\widehat{X}_{j}^{D_{3}}+K_{1} \widehat{Y}^{D_{3}}, \quad j=n, m, \\
\widehat{X}_{n}^{D_{3}}=\left[\frac{F_{n}^{(+)} F_{m}^{(+)} u_{n+1, m} u_{n-1, m}+\frac{1}{2}\left(F_{m}^{(-)}-F_{n}^{(-)} F_{m}^{(+)}\right) u_{n, m}\left(u_{n+1, m}+u_{n-1, m}\right)}{u_{n+1, m}-u_{n-1, m}}+\right. \\
\left.+\frac{F_{n}^{(-)} F_{m}^{(+)} u_{n, m}^{2}+\left(F_{m}^{(-)}-F_{n}^{(+)} F_{m}^{(+)}\right) u_{n, m}}{u_{n+1, m}-u_{n-1, m}}\right] \partial_{u_{n, m}},
\end{gathered}
$$


TABLE III. Identification of the coefficients of the symmetries of the ${ }_{i} D_{2}$ equations and value of the constants $K_{1}$ and $K_{2}$ in (16) in order to obtain non-autonomous YdKN equations.

\begin{tabular}{|c|c|c|c|c|c|c|c|c|c|}
\hline Eq. & $k$ & $a$ & $b_{k}$ & $c_{k}$ & $d$ & $e_{k}$ & $f$ & $K_{1}$ & $K_{2}$ \\
\hline \multirow[t]{2}{*}{${ }_{1} D_{2}$} & $n$ & 0 & 0 & 0 & 0 & $\frac{1}{2}\left[\delta_{1}\left(1+\delta_{2}\right)-1\right] F_{n}^{(+)} F_{m}^{(+)}+\frac{1}{2} F_{n}^{(-)} F_{m}^{(+)}-\frac{1}{2} F_{n}^{(-)} F_{m}^{(-)}$ & $-\delta_{2} F_{m}^{(-)}$ & 0 & $-1 / 2$ \\
\hline & $m$ & 0 & 0 & $-F_{n}^{(-)} F_{m}^{(+)} \delta_{1}$ & 0 & $\frac{1}{2}\left(\delta_{1}\left(1-\delta_{2}-1\right) F_{n}^{(-)} F_{m}^{(-)}-\frac{1}{2} F_{n}^{(+)} F_{m}^{(+)}-\frac{1}{2} \delta_{1} F_{n}^{(-)} F_{m}^{(+)}\right.$ & $\delta_{2}\left(\delta_{1}-1\right) F_{n}^{(-)}$ & 0 & $-1 / 2$ \\
\hline \multirow[t]{2}{*}{${ }_{2} D_{2}$} & $n$ & 0 & 0 & 0 & 0 & $\frac{1}{2}\left[1-\delta_{1}\left(1+\delta_{2}\right)\right] F_{n}^{(+)} F_{m}^{(-)}+\frac{1}{2} F_{n}^{(-)} F_{m}^{(-)}-\frac{1}{2} \delta_{1} F_{n}^{(-)} F_{m}^{(+)}$ & $\left(\delta_{1}-1\right) F_{m}^{(+)}$ & 0 & $-1 / 2$ \\
\hline & $m$ & 0 & 0 & $-\delta_{1} F_{n}^{(-)} F_{m}^{(-)}$ & 0 & $\frac{1}{2}\left[\delta_{1}\left(1-\delta_{2}\right)-1\right] F_{n}^{(-)} F_{m}^{(+)}-\frac{1}{2} F_{n}^{(+)} F_{m}^{(+)}-\frac{1}{2} \delta_{1} F_{n}^{(-)} F_{m}^{(+)}$ & $\delta_{2}\left[\delta_{1}-1\right] F_{n}^{(-)}+\lambda \delta_{1} F_{n}^{(+)}$ & 0 & $-1 / 2$ \\
\hline \multirow[t]{2}{*}{${ }_{3} D_{2}$} & $n$ & 0 & 0 & 0 & 0 & $\frac{1}{2}\left[\delta_{1}\left(1+\delta_{2}\right)-1\right] F_{n}^{(-)} F_{m}^{(-)}+\frac{1}{2} F_{n}^{(+)} F_{m}^{(-)}+\frac{1}{2} \delta_{1} F_{n}^{(-)} F_{m}^{(+)}$ & $\left(1-\delta_{1}\right) F_{m}^{(+)}$ & 0 & $1 / 2$ \\
\hline & $m$ & 0 & 0 & 0 & 0 & $\frac{1}{2}\left[\delta_{1}\left(1-\delta_{2}\right)-1\right] F_{n}^{(+)} F_{m}^{(+)}-\frac{1}{2} F_{n}^{(+)} F_{m}^{(-)}+\frac{1}{2} \delta_{1} F_{n}^{(-)} F_{m}^{(+)}$ & $\delta_{1} \lambda\left[-\delta_{1}\left(1+\delta_{2}\right)\right] F_{n}^{(+)}-\delta_{2} F_{n}^{(-)}$ & 0 & $1 / 2$ \\
\hline
\end{tabular}




$$
\begin{aligned}
\widehat{X}_{m}^{D_{3}} & =\left[\frac{F_{n}^{(+)} F_{m}^{(+)} u_{n, m+1} u_{n, m-1}+\frac{1}{2}\left(F_{n}^{(-)}-F_{n}^{(+)} F_{m}^{(-)}\right) u_{n, m}\left(u_{n, m+1}+u_{n, m-1}\right)}{u_{n, m+1}-u_{n, m-1}}+\right. \\
& \left.+\frac{F_{n}^{(+)} F_{m}^{(-)} u_{n, m}^{2}+\left(F_{n}^{(-)}-F_{n}^{(+)} F_{m}^{(+)}\right) u_{n, m}}{u_{n, m+1}-u_{n, m-1}}\right] \partial_{u_{n, m}}, \\
\hat{Y}^{D_{3}} & =\left[F_{n}^{(+)}\left(2 F_{m}^{(+)}+F_{m}^{(-)}\right)+F_{n}^{(-)}\right] u_{n, m} \partial_{u_{n, m}} .
\end{aligned}
$$

The two forms of $D_{4}$ possess only the following three-point generalized symmetries

$$
\begin{aligned}
\widehat{X}_{n}^{1} D_{4} & =\left[\frac{-\delta_{1} F_{n}^{(+)} u_{n+1, m} u_{n-1, m}-\frac{1}{2} u_{n, m}\left(u_{n+1, m}+u_{n-1, m}\right)}{u_{n+1, m}-u_{n-1, m}}+\right. \\
& \left.+\frac{-\delta_{1} F_{n}^{(-)} u_{n, m}^{2}+\delta_{2} \delta_{3} F_{m}^{(+)}}{u_{n+1, m}-u_{n-1, m}}\right] \partial_{u_{n, m}}, \\
\widehat{X}_{m}^{1} D_{4} & =\left[\frac{F_{m}^{(-)} u_{n, m+1} u_{n, m-1}+\frac{1}{2} u_{n, m}\left(u_{n, m+1}+u_{n, m-1}\right)}{u_{n, m+1}-u_{n, m-1}}+\right. \\
+ & \left.\frac{\delta_{2} F_{m}^{(+)} u_{n, m}^{2}-\delta_{1} \delta_{3} F_{n}^{(+)}}{u_{n, m+1}-u_{n, m-1}}\right] \partial_{u_{n, m}}, \\
\widehat{X}_{n}^{2} D_{4}= & {\left[\frac{-\delta_{1} \delta_{2} F_{n}^{(+)} F_{m}^{(+)} u_{n+1, m} u_{n-1, m}+\frac{1}{2} u_{n, m}\left(u_{n+1, m}+u_{n-1, m}\right)}{u_{n+1, m}-u_{n-1, m}}+\right.} \\
+ & \left.\frac{-\delta_{1} \delta_{2} F_{n}^{(-)} F_{m}^{(+)} u_{n, m}^{2}+\delta_{3}}{u_{n+1, m}-u_{n-1, m}}\right] \partial_{u_{n, m}}, \\
\widehat{X}_{m}^{2} D_{4} & =\left[\frac{\delta_{2} F_{n+m}^{(+)} u_{n, m+1} u_{n, m-1}+\frac{1}{2} u_{n, m}\left(u_{n, m+1}+u_{n, m-1}\right)}{u_{n, m+1}-u_{n+1}-u_{n, m-1} \delta_{3} F_{n}^{(+)}}+\partial_{u_{n, m}}\right.
\end{aligned}
$$

\begin{tabular}{|c|c|c|c|c|c|c|c|c|}
\hline Eq. & $k$ & $a$ & $b_{k}$ & $c_{k}$ & $d$ & $e_{k}$ & $f$ & $K_{1}$ \\
\hline \multirow[t]{2}{*}{$D_{3}$} & $n$ & 0 & 0 & $F_{n}^{(+)} F_{m}^{(+)}$ & 0 & $\frac{1}{2}\left(F_{n}^{(+)} F_{m}^{(-)}+F_{n}^{(-)} F_{m}^{(-)}-F_{n}^{(+)} F_{m}^{(+)}\right)$ & 0 & 0 \\
\hline & $m$ & 0 & 0 & $F_{n}^{(+)} F_{m}^{(+)}$ & 0 & $\frac{1}{2}\left(F_{n}^{(-)} F_{m}^{(+)}+F_{n}^{(-)} F_{m}^{(-)}-F_{n}^{(+)} F_{m}^{(+)}\right)$ & 0 & 0 \\
\hline \multirow[t]{2}{*}{${ }_{1} D_{4}$} & $n$ & 0 & 0 & $-\delta_{1}\left(F_{n}^{(+)} F_{m}^{(+)}+F_{n}^{(+)} F_{m}^{(-)}\right)$ & $-\frac{1}{2}$ & 0 & $\delta_{2} \delta_{3} F_{m}^{(+)}$ & - \\
\hline & $m$ & 0 & 0 & $\delta_{2}\left(F_{n}^{(+)} F_{m}^{(+)}+F_{n}^{(-)} F_{m}^{(+)}\right)$ & $\frac{1}{2}$ & 0 & $-\delta_{1} \delta_{3} F_{n}^{(+)}$ & - \\
\hline \multirow[t]{2}{*}{${ }_{2} D_{4}$} & $n$ & 0 & 0 & $-F_{n}^{(+)} F_{m}^{(+)} \delta_{1} \delta_{2}$ & $\frac{1}{2}$ & 0 & $\delta_{3}$ & - \\
\hline & $m$ & 0 & 0 & $\delta_{2}\left(F_{n}^{(+)} F_{m}^{(+)}+F_{n}^{(-)} F_{m}^{(-)}\right)$ & $\frac{1}{2}$ & 0 & $-\delta_{1} \delta_{3} F_{n}^{(+)}$ & - \\
\hline
\end{tabular}

but no point symmetries. Again the fluxes of the symmetries (20) can be readily identified with some specific form of the non-autonomous YdKN Equations (4) and the explicit form of the coefficients are shown in Table IV.

TABLE IV. Identification of the coefficients of the symmetries (20) for $D_{3},{ }_{1} D_{4}$, and ${ }_{2} D_{4}$ with those of a non-autonomous YdKN. 


\section{SYMMETRIES AND MASTER SYMMETRIES}

In Sec. II A-II C, we saw that the fluxes of all the one-point and three-point symmetries of the $H^{4}$ and $H^{6}$ equations are eventually related either to the YdKN (2) or to the non-autonomous YdKN Equation (4). In this section, we are interested in studying the integrability properties of these equations. Following the ideas in Ref. 23, we can use the method of the so-called master symmetries.

Master symmetries are a particular kind of evolution equations which depend on the field $u_{n, m}$, on the lattice variables, and/or on their evolution variable which can generate the whole hierarchy of symmetries of a given equation starting from a simple one. The notion of master symmetry has been introduced in Ref. 12, see also Refs. 11, 13, 14, and 27. Here we just present the method we can use to construct the master symmetries of the non-autonomous YdKN equation and its sub-cases following. $3,15,23,35$

Let us consider differential-difference equations of the Volterra type (1) and its generalized symmetry, possibly dependent on the lattice variable $n$ and on its symmetry variable $\tau$,

$$
u_{n, \tau}=\varphi_{n}\left(\tau, u_{n+k}, u_{n+k-1}, \ldots, u_{n+k^{\prime}+1}, u_{n+k^{\prime}}\right), \quad k^{\prime}<k .
$$

Let us define a Lie algebra structure on the set of functions $\varphi_{n}$ of the form (21). For any functions $\varphi_{n}^{(1)}$ and $\varphi_{n}^{(2)}$, we introduce the equations $u_{n, \tau_{1}}=\varphi_{n}^{(1)}$ and $u_{n, \tau_{2}}=\varphi_{n}^{(2)}$ and the corresponding total derivatives $D_{\tau_{i}}$ defined as

$$
D_{\tau_{i}}=\frac{\partial}{\partial \tau_{i}}+\sum_{j \in \mathbb{Z}} \varphi_{n+j}^{(i)} \frac{\partial}{\partial u_{n+j}}
$$

where $\varphi_{n}^{(1)}$ and $\varphi_{n}^{(2)}$ depend on different symmetry variables $\tau_{1}$ and $\tau_{2}$ and on $u_{\alpha}$ with $\alpha$ in $n+k_{1}$ $\geq \alpha \geq n+k_{1}^{\prime}$ and $n+k_{2} \geq \alpha \geq n k_{2}^{\prime}$, respectively. A new function is then defined by a Lie bracket [, ],

$$
\varphi_{n}^{(3)}=\left[\varphi_{n}^{(1)}, \varphi_{n}^{(2)}\right]=D_{\tau_{2}} \varphi_{n}^{(1)}-D_{\tau_{1}} \varphi_{n}^{(2)} .
$$

It is obviously skew-symmetric

$$
\left[\varphi_{n}^{(1)}, \varphi_{n}^{(2)}\right]=-\left[\varphi_{n}^{(2)}, \varphi_{n}^{(1)}\right],
$$

and as one can check by a direct calculation, it satisfies the Jacobi identity

$$
\left[\left[\varphi_{n}^{(1)}, \varphi_{n}^{(2)}\right], \varphi_{n}^{(3)}\right]=\left[\left[\varphi_{n}^{(1)}, \varphi_{n}^{(3)}\right], \varphi_{n}^{(2)}\right]+\left[\varphi_{n}^{(1)},\left[\varphi_{n}^{(2)}, \varphi_{n}^{(3)}\right]\right] .
$$

A differential-difference of the form

$$
u_{n, \tau}=g\left(u_{n+k}, u_{n+k-1}, \ldots, u_{n+k^{\prime}+1}, u_{n+k^{\prime}}\right), \quad k^{\prime}<k
$$

will be a generalized symmetry for a Volterra-like Equation (1) if its right hand side commutes with the right hand side of the Volterra-like equation, i.e., $\left[g_{n}, f_{n}\right]=0$. This generalized symmetry will reduce to a one-point symmetry if $k=k^{\prime}=0$ and it is called non-trivial if $k>1$ and $k^{\prime}<-1$.

A differential-difference (21) is called a master symmetry for a Volterra-like Equation (1) if the function

$$
g=\left[\varphi_{n}, f_{n}\right]
$$

is the right hand side of a nontrivial generalized symmetry. The function $\varphi_{n}$ satisfies then the following equation:

$$
\left[\left[\varphi_{n}, f_{n}\right], f_{n}\right]=0 .
$$

Eq. (23) has always the trivial solution: $\varphi_{n}=g_{n}$. The master symmetry corresponds to a nontrivial solution of (28).

A practical way of computing master symmetries of (1) is given by considering symmetry (21) of the form

$$
u_{n, \tau}=n f_{n}\left(u_{n+1}, u_{n}, u_{n-1}\right),
$$

where $f_{n}$ is the right hand side of (1). If $f_{n}$ depends on some constants, say $\alpha_{i}, i=1, \ldots, K$, we replace such constants by functions of the master symmetry variable $\tau$,

$$
\alpha_{i} \rightarrow \kappa_{i}=\kappa_{i}(\tau), \quad i=1, \ldots, M .
$$

Then we can impose the condition that symmetry (29) is actually a master symmetry, i.e., satisfies (23). Due to the definition of the total derivative $D_{\tau}(22)$, the annihilation will yield a set of first order 
differential equations for the new functions $\kappa_{i}(\tau)$ with the initial conditions given by the original value $\alpha_{i}$,

$$
\begin{gathered}
\kappa_{i}^{\prime}(\tau)=G_{i}\left(\kappa_{1}(\tau), \ldots, \kappa_{K}(\tau)\right), \quad i=1, \ldots, K, \\
\kappa_{i}(0)=\alpha_{i} .
\end{gathered}
$$

Then the symmetries for the original Equation (1) are obtained from the master symmetry (29) by putting $\tau=0$ in the resulting symmetry.

Let us construct the master symmetries of the non-autonomous YdKN Equation (4) as this differential difference equation appears in all symmetries of the generalized ABS equations we are considering. We proceed with the method we discussed above. Using the fact that $b_{k}, c_{k}$, and $e_{k}$ are two periodic, i.e.,

$$
b_{k}=b+(-1)^{k} \beta, \quad c_{k}=c+(-1)^{k} \gamma, \quad e_{k}=e+(-1)^{k} \eta,
$$

and substituting the coefficients $a, b, c, \gamma, d, e, \eta$, and $f$ with the function of $\tau$, we obtain the following expression for (5):

$$
\begin{aligned}
A_{k}\left(q_{k}, \tau\right)= & a(\tau) q_{k}^{2}+2\left[b(\tau)+(-1)^{k} k \beta(\tau)\right] q_{k} \\
+ & c(\tau)+(-1)^{k} \gamma(\tau), \\
B_{k}\left(q_{k}, \tau\right) & =\left[b(\tau)-(-1)^{k} \beta(\tau)\right] q_{k}^{2}+d(\tau) q_{k} \\
& +e(\tau)-(-1)^{k} \eta(\tau), \\
C_{k}\left(q_{k}, \tau\right) & =\left[c(\tau)-(-1)^{k} \gamma(\tau)\right] q_{k}^{2} \\
& +2\left[e(\tau)+(-1)^{k} \eta(\tau)\right] q_{k}+f(\tau),
\end{aligned}
$$

from which we may build up the $\tau$-dependent version of (4),

$$
q_{k t}=\frac{A_{k}\left(q_{k}, \tau\right) q_{k+1} q_{k-1}+B_{k}\left(q_{k}, \tau\right)\left(q_{k+1}+q_{k-1}\right)+C_{k}\left(q_{k}, \tau\right)}{q_{k+1}-q_{k-1}} .
$$

We then make ansatz (29) for the master symmetry, i.e.,

$$
q_{k \tau}=n \frac{A_{k}\left(q_{k}, \tau\right) q_{k+1} q_{k-1}+B_{k}\left(q_{k}, \tau\right)\left(q_{k+1}+q_{k-1}\right)+C_{k}\left(q_{k}, \tau\right)}{q_{k+1}-q_{k-1}} .
$$

Commuting the flows of $D_{t}$ and $D_{\tau}$, we obtain a five-point symmetry, $g_{n}^{(1)}$, according to (27). This obtained function has a purely three-point part which is of the same form (34) but with different coefficients. Annihilating this part, we obtain some equations for the coefficients $a, b, \beta, c, \gamma, d, e$, $\eta$, and $f$,

$$
\begin{aligned}
& a_{\tau}(\tau)=a(\tau) d(\tau)+2 \beta^{2}(\tau)-2 b^{2}(\tau), \\
& b_{\tau}(\tau)=-b(\tau) c(\tau)+\beta(\tau) \gamma(\tau)+a(\tau) e(\tau), \\
& \beta_{\tau}(\tau)=c(\tau) \beta(\tau)-b(\tau) \gamma(\tau)-a(\tau) \eta(\tau), \\
& c_{\tau}(\tau)=2 b(\tau) e(\tau)-d(\tau) c(\tau)-2 \beta(\tau) \eta(\tau), \\
& \gamma_{\tau}(\tau)=2 \beta(\tau) e(\tau)-d(\tau) \gamma(\tau)-2 \eta(\tau) b(\tau), \\
& d_{\tau}(\tau)=-c^{2}(\tau)+a(\tau) f(\tau)+\gamma^{2}(\tau), \\
& e_{\tau}(\tau)=-c(\tau) e(\tau)-\gamma(\tau) \eta(\tau)+b(\tau) f(\tau), \\
& \eta_{\tau}(\tau)=\gamma(\tau) e(\tau)-\beta(\tau) f(\tau)+c(\tau) \eta(\tau), \\
& f_{\tau}(\tau)=f(\tau) d(\tau)-2 e^{2}(\tau)+2 \eta^{2}(\tau)
\end{aligned}
$$


TABLE V. The value of the coefficients of master symmetry (35) obtained by solving system (37) in the case of the trapezoidal $H^{4}$ Equations (9).

\begin{tabular}{|c|c|c|c|c|}
\hline Eq. & Dir. & $c(\tau)$ & $\gamma(\tau)$ & $d(\tau)$ \\
\hline \multirow[t]{2}{*}{${ }_{t} H_{1}^{\varepsilon}$} & $n$ & $\frac{-1}{\tau+1}$ & 0 & $\frac{1}{\tau+1}$ \\
\hline & $m$ & $\frac{\varepsilon^{2}}{2}$ & $\frac{\varepsilon^{2}}{2}$ & 0 \\
\hline \multirow[t]{2}{*}{${ }_{t} H_{2}^{\varepsilon}$} & $n$ & $\frac{-1}{\tau+1}$ & 0 & $\frac{1}{\tau+1}$ \\
\hline & $m$ & $-\frac{\varepsilon}{2}$ & $-\frac{\varepsilon}{2}$ & 0 \\
\hline \multirow{3}{*}{${ }_{t} H_{3}^{\varepsilon}$} & & $\alpha_{2}^{2}\left(\alpha_{2}^{2}-1\right) e^{-\frac{1}{2} \tau \alpha_{2}\left(\alpha_{2}^{2}-1\right)}$ & & ${ }_{1} \alpha_{2}\left(1-\alpha_{2}^{2}\right)\left[e^{-\tau \alpha_{2}\left(\alpha_{2}^{2}-1\right)}+\alpha_{2}^{2}\right]$ \\
\hline & $n$ & $e^{-\tau \alpha_{2}\left(\alpha_{2}^{2}-1\right)}-\alpha_{2}^{2}$ & 0 & $\frac{1}{2}+e^{-\tau \alpha_{2}\left(\alpha_{2}^{2}-1\right)}-\alpha_{2}^{2}$ \\
\hline & $m$ & $-\frac{\varepsilon^{2} e^{-\frac{1}{2} \alpha_{3} \tau}}{2}$ & $-\frac{\varepsilon^{2} e^{-\frac{1}{2} \alpha_{3} \tau}}{2}$ & $\frac{\alpha_{3}}{2}$ \\
\hline Eq. & Dir. & $e(\tau)$ & $\eta(\tau)$ & $f(\tau)$ \\
\hline \multirow[t]{2}{*}{${ }_{t} H_{1}^{\varepsilon}$} & $n$ & 0 & 0 & $-\varepsilon^{2} \alpha_{2}^{2} F_{m}^{(+)}(\tau+1)$ \\
\hline & $m$ & 0 & 0 & 2 \\
\hline \multirow[t]{3}{*}{${ }_{t} H_{2}^{\varepsilon}$} & $n$ & $\varepsilon \alpha_{2}^{2} F_{m}^{(+)}(\tau+1)$ & 0 & $-\alpha_{2}^{2}\left[-1+\alpha_{2}^{2} F_{m}^{(+)}(\tau+1)^{2} \varepsilon^{2}+4 F_{m}^{(+)}\left(\alpha_{2}+2 \alpha_{3}\right) \varepsilon\right](\tau+1)$ \\
\hline & $m$ & $\frac{1}{2}+\frac{1}{4}\left(\tau-2 \alpha_{2}-2 \alpha_{3}\right) \varepsilon$ & $-\frac{1}{4}\left(\tau-2 \alpha_{2}-2 \alpha_{3}\right) \varepsilon$ & $-\frac{1}{4}\left(\tau-2 \alpha_{2}-2 \alpha_{3}\right)^{2} \varepsilon-\alpha_{3}-\frac{1}{2} \tau$ \\
\hline & & & & $\varepsilon^{2} \delta^{2} F_{m}^{(+)}\left(\alpha_{2}^{2}-1\right)\left(e^{-\tau \alpha_{2}\left(\alpha_{2}^{2}-1\right)}-\alpha_{2}^{2}\right)$ \\
\hline \multirow{2}{*}{${ }_{t} H_{3}^{\varepsilon}$} & $n$ & 0 & 0 & $e^{-1 / 2 \tau \alpha_{2}\left(\alpha_{2}^{2}-1\right)}$ \\
\hline & $m$ & 0 & 0 & $-\alpha_{3}^{2} \delta^{2} e^{\frac{1}{2} \alpha_{3} \tau}$ \\
\hline
\end{tabular}

If the coefficients satisfy system (36) then it is easy to show that the obtained $g_{n}^{(1)}$ is a generalized symmetry depending on five points. We remark that system (36) in its generality is impossible to solve, but since the right hand is a polynomial, we are ensured that such solution always exists in a neighbourhood of $\tau=0$.

The solutions with the initial conditions given by Tables I-IV will then yield explicit form of the master symmetries in all the relevant sub-cases. By using the master symmetry constructed above, we can construct infinite hierarchies of many-point generalized symmetries of the $H^{4}$ and $H^{6}$ equations in both directions. Furthermore, since for every $H^{4}$ and $H^{6}$ equation we have $a=b=\beta=0$, we can in fact use the reduced system where $a(\tau)=b(\tau)=\beta(\tau)=0$,

$$
\begin{aligned}
& c_{\tau}(\tau)=-d(\tau) c(\tau), \\
& \gamma_{\tau}(\tau)=-d(\tau) \gamma(\tau), \\
& d_{\tau}(\tau)=-c^{2}(\tau)+\gamma^{2}(\tau), \\
& e_{\tau}(\tau)=-c(\tau) e(\tau)-\gamma(\tau) \eta(\tau), \\
& \eta_{\tau}(\tau)=\gamma(\tau) e(\tau)+c(\tau) \eta(\tau), \\
& f_{\tau}(\tau)=f(\tau) d(\tau)-2 e^{2}(\tau)+2 \eta^{2}(\tau)
\end{aligned}
$$

The same comments made for (36) apply also to (37). As an example in Table V, we list the form of the $\tau$-dependent coefficients in the case of the trapezoidal $H^{4}$ Equations (9). We leave to the reader the construction of the master symmetries in the remaining simple cases.

\section{ALGEBRAIC ENTROPY FOR THE NON-AUTONOMOUS YDKN EQUATION AND ITS SUBCASES}

In Secs. II and III, we constructed the three-point generalized symmetries and master symmetries of the $H^{4}$ and $H^{6}$ equations which are eventually related either to YdKN (2) or to the non-autonomous YdKN Equation (4). 
It was remarked in the Introduction that the non-autonomous YdKN Equation (4) passes the necessary condition for the integrability which is an indication of the integrability of such class of equation. In this paper, we have shown that (4) are symmetries of the $H^{4}$ and $H^{6}$ equations. In this section, we give further evidence that the non-autonomous YdKN might be an integrable differentialdifference equation based on the algebraic entropy test ${ }^{5}$ for differential-difference equations.

We recall briefly how to compute the algebraic entropy ${ }^{9,30}$ in the case of differential-difference equations of the form

$$
\frac{\mathrm{d} u_{n}}{\mathrm{~d} t}=f_{n}\left(u_{n+1}, u_{n}, u_{n-1}\right) .
$$

First of all we assume that the equation is solvable for $u_{n+1}$ uniquely. This is a condition on $f_{n}$. Then, starting from the initial conditions at $n=-1,0$, we compute $u_{1}$ by substituting all relevant $t$ derivatives of $u_{-1}$ and $u_{0}$. Knowing $u_{1}$ we can then calculate $u_{2}$ and so on. Calculating the successive terms becomes rapidly very cumbersome if we consider the initial conditions to be independent and arbitrary. However a great simplification in the explicit calculations is obtained if instead of a generic initial condition one parameterizes the curve of the initial condition rationally using the variable $t$,

$$
u_{-1}=\frac{A_{-1} t+B_{-1}}{A t+B}, \quad u_{0}=\frac{A_{0} t+B_{0}}{A t+B} .
$$

We can then proceed and calculate $N$ iterates, for a sufficiently large positive integer $N$. At every passage $l$, we take the maximum of the degrees in $t$ between the numerator, $u_{l}^{\text {num }}$, and the denominator, $u_{l}^{\mathrm{den}}$

$$
d_{l}=\max \left\{u_{l}^{\mathrm{num}}, u_{l}^{\mathrm{den}}\right\},
$$

and, after $N$ iterates, we end up with the sequence of the degrees of the iterates

$$
1,1, d_{1}, d_{2}, \ldots, d_{N} .
$$

The next step is then extracting the value of the algebraic entropy which is defined as

$$
\eta=\lim _{l \rightarrow \infty} \frac{1}{l} \log d_{l} .
$$

This will in principle need the knowledge of the full sequence, but we know just the first $N$ terms. However, we have a standard heuristic method to extract such asymptotic quantity from the finite sequence (40): we can find a generating function of the sequence using rational functions

$$
g(s)=\sum_{l=0}^{\infty} d_{l} s^{l} .
$$

The method has already been shown to work remarkably well for maps and lattice equations ${ }^{16,29}$ and leads to extremely simple rational fractions with integer coefficients. This approximation can be used as a predictive tool. Indeed, one can readily compute the successive terms in the Taylor expansion for (42) and confront them with the degrees calculated with the iterations. This means that the assumption that the value of the algebraic entropy given by the approximate method is in fact very strong and very unlikely the real value will differ from it.

Having a rational generating function will also yield the value of the algebraic entropy from the modulus of the smallest pole of the generating function

$$
\eta=\log \min \left\{|s|\left|\lim _{\sigma \rightarrow s}\right| g(\sigma) \mid=\infty\right\} .
$$

From the generating function, one can also find an asymptotic fit for degrees (39). This can be done by using the $\mathcal{Z}$-transform ${ }^{10,21}$ because it can be readily proved that

$$
d_{n}=\mathcal{Z}\left[g\left(\frac{1}{\zeta}\right)\right]_{n},
$$

where $\mathcal{Z}[f(\zeta)]_{n}$ is the $\mathcal{Z}$-transform of the function $f(\zeta)$. For more details on how the method is implemented see Ref. 17. 
We look for the sequence of degrees of the iterate map for the non-autonomous YdKN Equation (4) and its particular cases found in Sec. II. We find for all the cases, except the symmetries of ${ }_{r} H_{1}^{\varepsilon}$, i.e., for symmetries (12) and (14) of ${ }_{t} H_{1}^{\varepsilon}$ and for the symmetries of the ${ }_{i} D_{2}$ equations, the following values:

$$
1,1,3,7,13,21,31,43,57,73,91,111,133,157 \ldots
$$

This sequence has the following generating function:

$$
g(z)=\frac{1-2 z+3 z^{2}}{(1-z)^{2},}
$$

which gives the following quadratic fit for sequence (45),

$$
d_{l}=l(l-1)+1,
$$

therefore, the algebraic entropy is zero.

For the symmetry in the $n$ direction (6a) of the equation ${ }_{r} H_{1}^{\varepsilon}$, we have the somehow different situation that the sequence growth is different depending on whether we consider the even or odd values of the $m$ variable

$$
\begin{gathered}
m=2 k \quad 1,1,3,7,10,17,23,33,42,55,67,83,98,117 \ldots, \\
m=2 k+1 \quad 1,1,3,4,9,13,21,28,39,49,63,76,93,109 \ldots .
\end{gathered}
$$

These sequences have the following generating functions and asymptotic fits:

$$
\begin{array}{r}
g(z)=\frac{2 z^{5}-3 z^{4}+3 z^{3}+z^{2}-z+1}{(1-z)^{3}(z+1)}, \\
d_{l}=\frac{3}{4} l^{2}-l-\frac{5(-1)^{l}-21}{8}, \\
\quad g(z)=\frac{\left(z^{2}+z+1\right)\left(2 z^{2}-2 z+1\right)}{(1-z)^{3}(z+1)}, \\
m=2 k+1, \quad d_{l}=\frac{3}{4} l^{2}-\frac{3}{2} l-\frac{5(-1)^{l}-19}{8} .
\end{array}
$$

The symmetry in the $m$ direction (8b) of the equation ${ }_{r} H_{1}^{\varepsilon}$ has the same behaviour obtained by exchanging $m$ with $n$ in formulae (48) and (49).

The symmetry of the equation ${ }_{t} H_{1}^{\varepsilon}$ in the $n$ direction has almost the same growth as that obtained for $m$ odd (48b) and (49b); however, the fit $d_{l}=\frac{3}{4} l^{2}-\frac{5}{4} l+(-1)^{l} \frac{l}{4}+\frac{(-1)^{l}+15}{8}$ presents a term $l(-1)^{l}$, new in this kind of results. For $m$ even, we have the same growth as (45). The symmetry of the equation ${ }_{t} H_{1}^{\varepsilon}$ in the $m$ direction has the same growth as the even one of ${ }_{t} H_{1}^{\varepsilon}$ (48a) and (49a). For symmetries (16) of the ${ }_{i} D_{2}$ equation, we have different growth according to the even or odd values of the $m$ and $n$ variables. These sequences are slightly lower than in the case of equations $H_{1}^{\varepsilon}$, however, always corresponding to a quadratic asymptotic fit.

This shows that the whole family of the non-autonomous YdKN is integrable according to the algebraic entropy test. For completeness, let us just mention that symmetries (17) of the ${ }_{i} D_{2}$ equations have a sequence growth of the same order than those considered above, i.e., quadratic growth and thus null entropy.

Let us show the validity of the algebraic entropy test by the calculation of a symmetry for a non-integrable subcase of ${ }_{t} H_{1}^{\varepsilon}$. As the symmetries of the ${ }_{t} H_{1}^{\varepsilon}$ equation depend on arbitrary functions, not all of them will produce an integrable flux. Let us consider the case of flux (11) when $\varepsilon=0, B_{n}(x)$ $=-1 / x, \gamma_{m}=\delta_{m}=0$, and $\alpha=1$. We have the following symmetry:

$$
\widehat{X}_{n}^{P}=\frac{u_{n+1, m} u_{n-1, m}-u_{n, m}^{2}}{u_{n+1, m}-2 u_{n, m}+u_{n-1, m}} \partial_{u_{n, m}} .
$$


Following Ref. 35, the necessary condition for the flux of (50) $\frac{d u_{n, m}}{d t}=\widehat{X}_{n}^{P} u_{n, m}$ to be integrable is that given

$$
p_{1}=\log \frac{\partial f_{n}}{\partial u_{n+1}}=2 \log \left(\frac{u_{n, m}-u_{n-1, m}}{u_{n+1, m}-2 u_{n, m}+u_{n-1, m}}\right) \text {, }
$$

we must have

$$
\begin{aligned}
\frac{\mathrm{d} p_{1}}{\mathrm{~d} t} & =\frac{-2 u_{n, m}^{2}+4 u_{n, m} u_{n+1, m}-2 u_{n+1, m}^{2}}{\left(u_{n+2, m}-2 u_{n+1, m}+u_{n, m}\right)\left(-u_{n+1, m}+2 u_{n, m}-u_{n-1, m}\right)} \\
& -\frac{2\left(u_{n-1, m}-u_{n+1, m}\right)\left(-u_{n+1, m}+u_{n, m}\right)}{\left(u_{n+1, m}-2 u_{n, m}+u_{n-1, m}\right)^{2}} \\
& +\frac{2 u_{n, m}^{2}-2 u_{n, m} u_{n+1, m}-2 u_{n-1, m} u_{n, m}+2 u_{n-1, m} u_{n+1, m}}{\left(u_{n, m}-2 u_{n-1, m}+u_{n-2, m}\right)\left(-u_{n+1, m}+2 u_{n, m}-u_{n-1, m}\right)} \\
& =(T-1) g_{n}
\end{aligned}
$$

for any function $g_{n}$ defined on a finite portion of the lattice, for example, such that $g_{n}=g_{n}\left(u_{n+1, m}, u_{n, m}\right.$, $\left.u_{n-1, m}, u_{n-2, m}\right)$. We search the function $g_{n}$ using the partial sum method ${ }^{35}$ and we find an obstruction at the third passage. Then the function $g_{n}$ does not exists and therefore we conclude that the flux of (50) is a non-integrable differential-difference equation.

Using the algebraic entropy test on the flux of (50), we find the following values for the degrees of the iterates:

$$
1,1,3,9,27,81,273,729 \ldots
$$

which gives us the following generating function

$$
g(s)=\frac{1-2 s}{1-3 s},
$$

and the entropy is clearly nonvanishing $\eta=\log 3$. So the non-integrability result obtained by the approximate algebraic entropy method agrees with those obtained by applying the formal generalized symmetry method.

\section{CONCLUSIONS}

In this note, we constructed the symmetries of the equations belonging to the Boll classification ${ }^{7,8}$ and showed that they are integrable (by the algebraic entropy test) and related to particular cases of the non-autonomous YdKN Equation (4). ${ }^{26}$ This was already known for the rhombic $H^{4}$ equations ${ }^{32}$ and here we show the explicit identification of the symmetries obtained in that paper with the coefficients of the non-autonomous YdKN equation.

We finally note that, as was proved in Ref. 23 for YdKN (2), no equation belonging to the Boll classification has a symmetry which corresponds to the general non-autonomous YdKN Equation (4). In all the cases of the Boll classification, one has $a=b_{k}=0$.

In Ref. 31, it was shown that the $Q_{V}$ equation introduced by Viallet

$$
\begin{aligned}
Q_{V} & : p_{4}+p_{3}\left(u_{n, m}+u_{n, m+1}+u_{n+1, m}+u_{n+1, m+1}\right)+ \\
& +p_{2,1}\left(u_{n, m} u_{n+1, m}+u_{n, m+1} u_{n+1, m+1}\right)+ \\
& +p_{2,2}\left(u_{n, m} u_{n, m+1}+u_{n+1, m} u_{n+1, m+1}\right)+ \\
& +p_{2,0}\left(u_{n, m} u_{n+1, m+1}+u_{n, m+1} u_{n+1, m}\right)+ \\
& +p_{I}\left(u_{n, m} u_{n, m+1} u_{n+1, m}+u_{n, m} u_{n+1, m} u_{n+1, m+1}+\right. \\
& \left.+u_{n, m} u_{n, m+1} u_{n+1, m+1}+u_{n, m+1} u_{n+1, m} u_{n+1, m+1}\right)+ \\
& +p_{0} u_{n, m} u_{n, m+1} u_{n+1, m} u_{n+1, m+1}=0
\end{aligned}
$$

possesses the Klein symmetry and admits a symmetry of the form of the YdKN 


$$
\widehat{X}_{n}^{\mathrm{V}}=\frac{h}{u_{n+1, m}-u_{n-1, m}}-\frac{1}{2} \partial_{u_{n+1, m}} h,
$$

where

$$
\begin{aligned}
h\left(u_{n, m}, u_{n+1, m} ; p_{1}, p_{2, i}, p_{3}, p_{4}\right)= & Q_{V} \partial_{u_{n, m+1}} \partial_{u_{n+1, m+1}} Q_{V}+ \\
& -\left(\partial_{u_{n, m+1}} Q_{V}\right)\left(\partial_{u_{n+1, m+1}} Q_{V}\right) .
\end{aligned}
$$

The connection formulae between the coefficient of $Q_{V}$ and YdKN (2) are

$$
\begin{aligned}
& a=p_{1}^{2}-p_{2,1} p_{0}, \quad b=\frac{1}{2}\left[p_{1}\left(p_{2,0}+p_{2,2}-p_{2,1}\right)-p_{3} p_{0}\right], \\
& c=p_{2,0} p_{2,2}-p_{3} p_{1}, \quad d=\frac{1}{2}\left[p_{2,2}^{2}-p_{2,1}^{2}+p_{2,0}^{2}-p_{0} p_{4}\right], \\
& e=\frac{1}{2}\left[p_{3}\left(p_{2,2}-p_{2,1}+p_{2,0}\right)-p_{1} p_{4}\right], \quad f=p_{3}^{2}-p_{2,1} p_{4} .
\end{aligned}
$$

This is a set of coupled nonlinear algebraic equations between the 7 parameters $p_{i}$ of $Q_{V}$ and the 6 ones $(a, \ldots, f)$ of the YdKN. Eq. (58) tells us that the YdKN with coefficients given by (58) is a three-point generalized symmetry of $Q_{V}$. If a solution of (58) exists, i.e., one is able to express the $p_{i}$ in terms of $(a, \cdots, f)$, then $Q_{V}$, maybe after a reparameterization, turns out to be a Bäcklund transformation of the YdKN. ${ }^{4,22}$

From the results obtained in this paper, one is lead to conjecture a non-autonomous generalization of the $Q_{V}$ equation. We have many possible ways of proposing such a generalization. A first possibility is to generalize the original Klein symmetry

$$
\begin{aligned}
& Q\left(u_{n+1, m}, u_{n, m}, u_{n+1, m+1}, u_{n, m+1} ;-(-1)^{n},(-1)^{m}\right)= \\
& \tau Q\left(u_{n, m}, u_{n+1, m}, u_{n, m+1}, u_{n+1, m+1} ;(-1)^{n},(-1)^{m}\right), \\
& Q\left(u_{n, m+1}, u_{n+1, m+1}, u_{n, m}, u_{n, m+1} ;(-1)^{n},-(-1)^{m}\right)= \\
& \tau^{\prime} Q\left(u_{n, m}, u_{n+1, m}, u_{n, m+1}, u_{n+1, m+1} ;(-1)^{n},(-1)^{m}\right),
\end{aligned}
$$

where $\left(\tau, \tau^{\prime}\right)= \pm 1$ and $Q\left(x, u, y, z,(-1)^{n},(-1)^{m}\right)$ is a multilinear function of its arguments with nonautonomous coefficients in the form of 2-periodic functions in $n$ and $m$, i.e., of the form $\alpha+\beta(-1)^{n}+$ $\gamma(-1)^{m}+\delta(-1)^{n+m}$, with $\alpha, \beta, \gamma$, and $\delta$ constants. This discrete symmetry is shared by all of the Boll systems and in the autonomous case reduces to the usual Klein symmetry.

A second possibility is to ask the function $Q\left(x, u, y, z ;(-1)^{n},(-1)^{m}\right)$ to respect a strict Klein symmetry just as in (55). Choosing the coefficients, for example, as

$$
\begin{gathered}
p_{0}=1+(-1)^{n}, p_{1}=(-1)^{n}, p_{2,1}=-1+(-1)^{n}, p_{2,2}=(-1)^{n}, \\
p_{2,0}=1+2(-1)^{n}, p_{3}=1+(-1)^{n}, p_{4}=4+2(-1)^{n},
\end{gathered}
$$

(58) provides a non-autonomous YdKN. In this case, by performing the algebraic entropy test the equation turns out to be integrable. Its generalized symmetries, however, are not necessarily in the form of a non-autonomous YdKN equation. A different non-autonomous choice of the coefficients of (55), such that (58) is satisfied for the coefficients of the non-autonomous YdKN, gives, by the algebraic entropy test, a non-integrable equation.

The proof of the existence of a non-autonomous generalization of $Q_{V}$ together with the derivation of an effective Bäcklund transformation and Lax pair for the YdKN and its non-autonomous counterpart is work in progress.

\section{ACKNOWLEDGMENTS}

C.S. and D.L. have been partly supported by the Italian Ministry of Education and Research, 2010 PRIN Continuous and discrete nonlinear integrable evolutions: from water waves to symplectic maps.

All the authors are supported by INFN IS-CSN4 Mathematical Methods of Nonlinear Physics. 
${ }^{1}$ Adler, V. E., Bobenko, A. I., and Suris, Y. B., "Classification of integrable equations on quad-graphs. The consistency approach," Commun. Math. Phys. 233, 513-543 (2003).

2 Adler, V. E., Bobenko, A. I., and Suris, Y. B., "Discrete nonlinear hyperbolic equations. Classification of integrable cases," Funct. Anal. Appl. 43, 3-17 (2009).

${ }^{3}$ Adler, V. E., Shabat, A. B., and Yamilov, R. I., "Symmetry approach to the integrability problem," Theor. Math. Phys. 125(3), 1603-1661 (2000).

${ }^{4}$ Adler, V. E. and Suris, Yu. B., “Q4: Integrable master equation related to an elliptic curve,” Int. Math. Res. Not. 2004(47), 2523-2553; e-print arXiv:nlin/0309030.

${ }^{5}$ Bellon, M. and Viallet, C-M., "Algebraic entropy," Commun. Math. Phys. 204, 425-437 (1999).

${ }^{6}$ Boll, R., "Classification of 3D consistent quad-equations," J. Nonlinear Math. Phys. 18, 337-365 (2011).

${ }^{7}$ Boll, R., "Classification and Lagrangian structure of 3D consistent quad-equations," Ph.D. dissertation (Technical University Berlin, 2012)

${ }^{8}$ Boll, R., "Corrigendum: Classification of 3D consistent quad-equations," J. Nonlinear Math. Phys. 19, 618-620 (2012).

${ }^{9}$ Demskoy, D. K. and Viallet, C.-M., “Algebraic entropy for semi-discrete equations,” J. Phys. A: Math. Theor. 45, 352001 (2012).

${ }^{10}$ Elaydi, S., An Introduction to Difference Equations, 3rd ed. (Springer, 2005).

${ }^{11}$ Fokas, A. S., "Symmetries and integrability," Stud. Appl. Math. 77, 253-299 (1987).

${ }^{12}$ Fokas, A. S. and Fuchssteiner, B., "The hierarchy of the Benjamin-Ono equation," Phys. Lett. A 86, 341-345 (1981).

${ }^{13}$ Fuchssteiner, B., "Master symmetries and higher order time-dependent symmetries and conserved densities of nonlinear evolution equations," Prog. Theor. Phys. 70, 1508-1522 (1983).

${ }^{14}$ Fuchssteiner, B., "On the hierarchy of the Landau-Lifshitz equation," Physica D 13, 387-394 (1984).

${ }^{15}$ Garifullin, R. N., Mikhailov, A. V., and Yamilov, R. I., "Discrete equation on a square lattice with a nonstandard structure of generalized symmetries," Theor. Math. Phys. 180(1), 765-780 (2014).

${ }^{16}$ Grammaticos, B., Halburd, R. G., Ramani, A., and Viallet, C.-M., "How to detect the integrability of discrete systems," J. Phys. A: Math. Theor. 42, 454002 (2009), Newton Institute Preprint NI09060-DIS.

${ }^{17}$ Gubbiotti, G., Ph.D. dissertation (2016).

${ }^{18}$ Gubbiotti, G., Levi, D., and Scimiterna, C., "On partial differential and difference equations with symmetries depending on arbitrary functions," Acta Polytecnica 56, 193-201 (2016).

${ }^{19}$ Gubbiotti, G., Scimiterna, C., and Levi, D., "Algebraic entropy, symmetries and linearization of quad equations consistent on the cube," J. Nonlinear Math. Phys. 23(4), 507-543 (2016).

${ }^{20}$ Gubbiotti, G., Scimiterna, C., and Levi, D., "Linearizability and fake Lax pair for a consistent around the cube nonlinear non-autonomous quad-graph equation,” Teor. Mat. Fiz. 189, 1459-1471 (2016).

${ }^{21}$ Jury, E., Theory and Applications of the Z-Transform Method (Robert E. Krieger, 1964).

${ }^{22}$ Levi, D., "Nonlinear differential-difference equations as Bäcklund transformations," J. Phys. A: Math. Gen. 14, 1083-1098 (1981).

${ }^{23}$ Levi, D., Petrera, M., Scimiterna, C., and Yamilov, R. I., "On Miura transformations and Volterra-type equations associated with the Adler-Bobenko-Suris equations," SIGMA 4, 077 (2008).

${ }^{24}$ Levi, D., Winternitz, P., and Yamilov, R. I., "Symmetries of the continuous and discrete Krichever-Novikov equation," SIGMA7, 097 (2011).

${ }^{25}$ Levi, D. and Yamilov, R. I., "Classification of evolutionary equations on the lattice I. The general theory," e-print arXiv:solvint/9511006 v1 (1995).

${ }^{26}$ Levi, D. and Yamilov, R. I., "Conditions for the existence of higher symmetries of the evolutionary equations on the lattice," J. Math. Phys. 38, 6648-6674 (1997).

${ }^{27}$ Oevel, W. and Fuchssteiner, B., "Explicit formulas for the symmetries and conservation laws of the Kadomtsev-Petviashvili equation," Phys. Lett. A 88, 323-327 (1982).

${ }^{28}$ Rasin, O. G. and Hydon, P. E., "Symmetries of integrable difference equations on the quad-graph,” Stud. Appl. Math. 119 253-269 (2007).

${ }^{29}$ Viallet, C. M., "Algebraic entropy for lattice equations," e-print arXiv:math-ph/0609043 (2006).

${ }^{30}$ Viallet, C. M., "Algebraic entropy for differential-delay equations," e-print arXiv:1408.6161 (2014).

${ }^{31}$ Xenitidis, P. D., "Integrability and symmetries of difference equations: The Adler-Bobenko-Suris case," in Proceedings of the 4th Workshop Group Analysis of Differential Equations and Integrable Systems (Institute of Mathematics of National Academy of Sciences of Ukraine, 2009), pp. 226-242; e-print arXiv:0902.3954.

${ }^{32}$ Xenitidis, P. D. and Papageorgiou, V. G., "Symmetries and integrability of discrete equations defined on a black and white lattice," J. Phys. A: Math. Theor. 42, 454025 (2009).

${ }^{33}$ Yamilov, R. I., "Classification of discrete evolution equations," Usp. Mat. Nauk 38, 155-156, (1983) (in Russian).

${ }^{34}$ Yamilov, R. I., "Discrete equations of the form $\dot{u}_{n}=F\left(u_{n-1}, u_{n}, u_{n+1}\right) n \in Z$ with an infinite number of local conservation laws," Ph.D. dissertation (Ufa: Soviet Academy of Sciences, 1984) (in Russian).

${ }^{35}$ Yamilov, R. I., "Symmetries as integrability criteria for differential difference equations," J. Phys. A: Math. Gen. 39, R541-R623 (2006). 